\title{
Comparison of Performance Parameters of Reactive, Proactive and Hybrid Routing Protocols in RWP Mobility Model through Qualnet
}

\author{
Ashish Allen Roberts and Ashish Xavier Das
}

\begin{abstract}
Wireless Sensors are devices that monitor and record their surroundings physical and environmental parameters and transmit them continuously to one of the source sensors. A collection of such wireless sensors form a network called Wireless Sensor Networks. This technology is considered to be the best for the study of performance parameters. Routing is a technique which we use to select path to send traffic in the network, also called as Protocol. AODV, DYMO, OLSR and IERP are adhoc routing protocols. In this paper we shall study different performance parameters in a Random Waypoint Mobility Model by varying the number of nodes and also changing the maximum speed of a node, such as Average Throughput, Average End to End Delay, Average Jitter, Average PDR (Packet Delivery Ratio) and Total Packets Received. Analysis and performance study is done using Qualnet 6.1 simulator.
\end{abstract}

Index Terms-Mobility model, performance parameters, qualnet, routing protocols.

\section{INTRODUCTION}

Wireless sensor networks (WSN) is a special class of adhoc wireless network that are used to provide a wireless communication infrastructure, observe and respond to phenomena in the natural environment and in our physical and cyber infrastructure [1]. A routing protocol is such that it specifies how routers communicate with one another, broadcast information that allows them to select their path between any two given nodes on an adhoc network.

A routing protocol distribute this information first among to its neighbours, and then to the whole of the network. In this way, the routers achieve knowledge of the topology of the network. An ad hoc routing protocol is a principle, or standard, that manages how the nodes decide which way it has to send packets between the communicating devices in the network. Some examples of routing protocols available for Ad- hoc networks are AODV, CGSR, DSDV, DSR, OLSR, WRP, ZRP etc. [1]. AODV and DYMO are also known as Reactive Protocols, whereas OLSR is a Proactive Protocol and IERP is a Hybrid Protocol [1]. Mobility model represent the movement of nodes and how their location, velocity and acceleration change with respect to time. In the study of a new Mobile ad hoc network protocol, it is important to simulate the protocol and evaluate its protocol performance.

Manuscript received February 21, 2013; revised April 27, 2013.

The authors are with the Sam Higginbottom Institute of Agriculture, Technology and Sciences (SHIATS-DU), Allahabad 211007, U. P. India (e-mail: ashualie_aai@yahoo.co.in, xavierashish@gmail.com).
Protocol simulation has several key parameters; including mobility model and communicating traffic pattern Mobility models characterize user-movement-patterns. Traffic models describe the condition of the mobile services [2].

\section{RANDOM WAYPOINT MOBILITY MODEL}

Random waypoint model is a random model for the movement of mobile users, and how their location, velocity and acceleration change over time. Mobility models are used for simulation purposes when new network protocols are evaluated. The Random waypoint model was first proposed by Johnson and Maltz [3]. It is one of the most popular mobility models and the "benchmark" mobility model to evaluate other Mobile ad hoc network (MANET) routing protocols, because of its simplicity and wide availability.

In random waypoint mobility model, the nodes randomly selects a position, moves towards it in a straight line at a constant speed that is randomly selected from a range, and pauses at that destination. The node repeats this, throughout the simulation.

\section{RouTING PROTOCOLS}

A lot of protocols have been designed for Ad-hoc networks since last few years. Routing protocols are a set of rules that a network adopts for movement of data packets in a timely and secured fashion [4]. Broadly routing protocols are classified into three categories: Reactive, Proactive and Hybrid. Proactive routing protocols are table driven protocols, when there is a need of data transfer the source node reached the path immediately which in turn helps in minimizing the bandwidth overhead and less time for the data packet movement. Proactive routing protocol used in our scenario is OLSR. Reactive protocols are on demand protocols, it finds a path within a network only when it is necessary [5]. Reactive protocols used in our scenario are AODV and DYMO. Hybrid protocol includes combination of Reactive and Proactive Routing Protocols. Hybrid protocol used in our scenario is IERP. The details of the routing protocols are mentioned below:

\section{A. AODV (Adhoc on Demand Distance Vector)}

Ad hoc On-demand Distance Vector Routing (AODV) [4] protocol is an on demand routing protocol [3] as it determines a route to the destination only when a node wants to send data to that destination. The source node broadcasts a route request (RREQ) packet when it wants to find path to the destination. 
The neighbors in turn broadcast the packet to their neighbors until it reaches a transitional node that has recent route information about the destination or until it reaches the destination A node records the node from which request packet received first to erect the reverse path for route reply to source node. As the route reply packet traverses back to the source, the nodes along the path enter the forward route into their tables. Due to the mobile nature of nodes, route maintenance is required. If the source moves then it can reinitiate route discovery to the destination. If one of the intermediate nodes move then moved nodes neighbor realizes the link failure and sends a link failure notification to its upstream neighbors and so on until it reaches the source upon which the source can reinitiate route discovery if needed. AODV [6] has greatly reduced the number of routing messages in the network.

\section{B. DYMO (Dynamic Mannet on Demand)}

Dynamic MANET On-demand (DYMO) routing protocol enables reactive, multi-hop unicast routing between participating DYMO routers. DYMO operation is split into route discovery and route maintenance. Routes are discovered on- demand when the originator initiates hop-by-hop distribution of a RREQ (rout request) message throughout the network to find a route to the target, currently not in its routing table. This RREQ message is swamped in the network using broadcast and the packet reaches its destination. The target then sends a RREP (route reply) to the source. Upon receiving the RREP message by the source, routes have been established between the two nodes. For maintenance of routes which are in use, routers elongate route lifetimes upon successfully forwarding a packet. In order to react to changes in the network topology, routers monitor links over which traffic is flowing [4]. DYMO uses sequence numbers to ensure loop freedom and enable them to determine the order of DYMO route discovery messages, thus avoiding use of outdated routing information [7].

\section{OLSR (Optimized Link State Routing)}

Optimized Link State Routing (OLSR) is a proactive MANET routing protocol. Unlike DSDV and AODV, OLSR reduces the number of retransmissions by providing optimal routes in terms of number of hops. Only the MPRs of a node retransmit its broadcast messages, hence no extra control traffic is generated in response to link failures. OLSR is particularly suitable for large and dense networks. The path from source to destination consists of a sequence of hops through the MPRs. In OLSR, a HELLO message is broadcasted to all of its neighbors containing information about its neighbors and their link status and received by the nodes which are one hop away but they are not passed on to further nodes [4]. In response of HELLO messages, each node would construct its MPR Selector table. MPRs of a given node are declared in the subsequent HELLO messages transmitted by this node

\section{IERP (Interzone Routing Protocol)}

Interzone Routing Protocol (IERP), the reactive routing component of the Zone Routing Protocol (ZRP). IERP adapts existing reactive routing protocol implementations to take advantage of the known topology of each node's surrounding r-hop neighborhood (routing zone), provided by the Intrazone Routing Protocol (IARP). The availability of routing zone routes allows IERP to suppress route queries for local destinations [8]. When a global route discovery is required, the routing zone based bordercast service can be used to efficiently guide route queries outward, rather than blindly relaying queries from neighbor to neighbor.

\section{SCENARIO}

The simulator used to record the performance parameters is Qualnet 6.1 developed by SCALABLE Network Technologies. In the Architecture mode of the simulator the scenario is designed in an area of 360000 square meters. Number of nodes is kept as 20. Network traffic type is chosen as CBR (Constant Bit Rate) type, we have used 2 CBRs, where 1 CBR connects 2 nodes. The time for which the simulation is performed is 600 seconds. The node mobility model is set up as Random Waypoint Mobility, and further the minimum speed of the nodes at which they move randomly is set as $1 \mathrm{~m} / \mathrm{s}$ and maximum speed of the nodes is set as $20 \mathrm{~m} / \mathrm{s}$, this, and the pause time is set as $30 \mathrm{~ms}$. A total of 100 data packets are sent over the $2 \mathrm{CBR}$ traffic with an individual payload of 512 bytes. Initially the routing protocol is set as AODV and the option of add to batch is used to compare this simulation data with other routing protocols, DYMO, OLSR and IERP (see Table I).

TABLE I: SCENARIO DESCRIPTION

\begin{tabular}{|l|c|}
\hline \multicolumn{1}{|c|}{ PARAMETERS } & VALUES \\
\hline Simulator & QUALNET 6.1 \\
\hline Protocols studied & AODV,DYMO,OLSR \& IERP \\
\hline Number of nodes & 20 nodes \\
\hline Simulation time & $600 \mathrm{~s}$ \\
\hline Simulation area & $600 * 600 \mathrm{sq} \mathrm{m}$ \\
\hline Node movement model & Random waypoint mobility \\
\hline Traffic types & $2 \mathrm{CBR}$ sources \\
\hline Mobility of nodes & Min speed $=1 \mathrm{~m} / \mathrm{s}$, Max speed $=10 \mathrm{~m} / \mathrm{s}$ \\
\hline
\end{tabular}

Below are the screenshots from the Qualnet simulator when the above mentioned scenario is designed in the architect mode and before the results are analyzed through the analyzer, the Qualnet software runs the experiment.

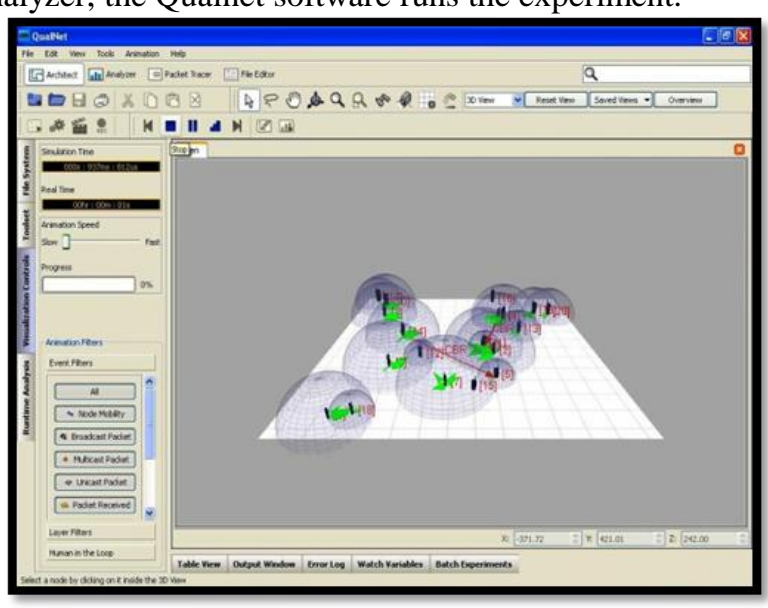

Fig. 1. 3D view of scenario simulation in Qualnet. 


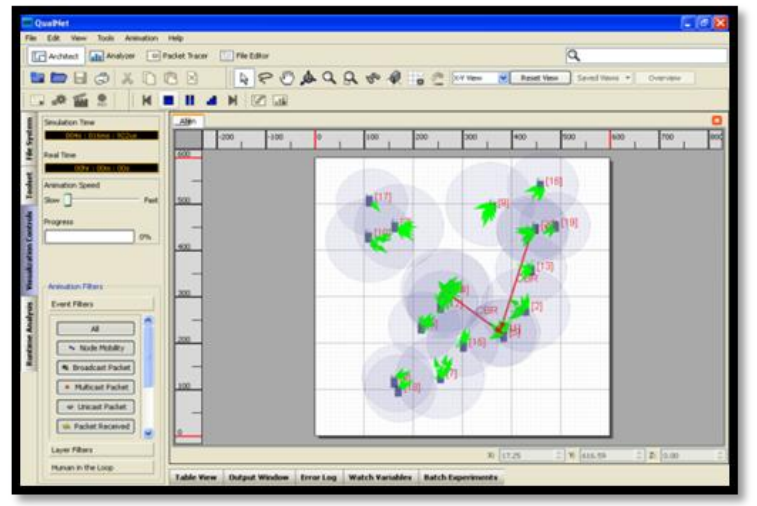

Fig. 2. X-Y view of simulation setup.

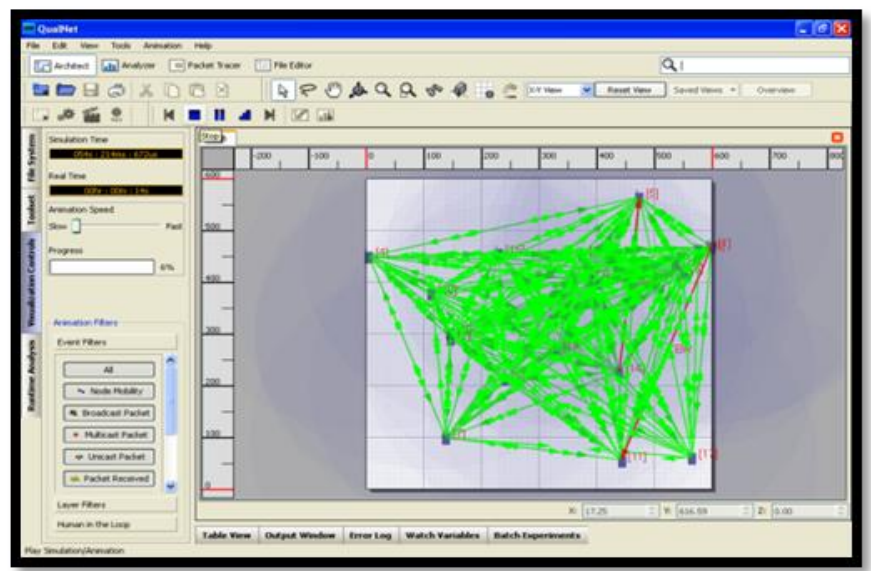

Fig. 3. Broadcasting and network setup of protocols.

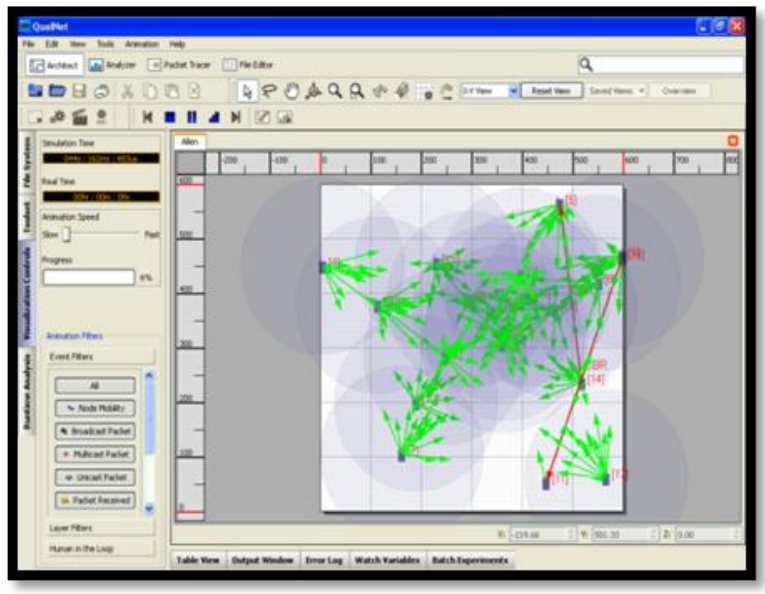

Fig. 4. Random waypoint mobility and CBR connections.

\section{RESUlTS AND DISCUSSION}

\section{A. Throughput}

In a mobile or data communication network throughput is the average rate of successful message delivery in a communication channel. This data may be delivered over a physical or logical link, or pass through a certain network node. The throughput is usually measured in bits per second (bit/s or bps), and sometimes in data packets per second or data packets per time slot. [3], [4]. Better the throughput better will be the communication system. Here the graph shows that we have a better throughput in DYMO and AODV in comparison to OLSR and IERP. In routing protocols
DYMO and AODV, the performance parameter: throughput exhibits better results due to its path finding techniques, which uses the least and secure path in its network as compared to the other routing protocols OLSR and IERP.

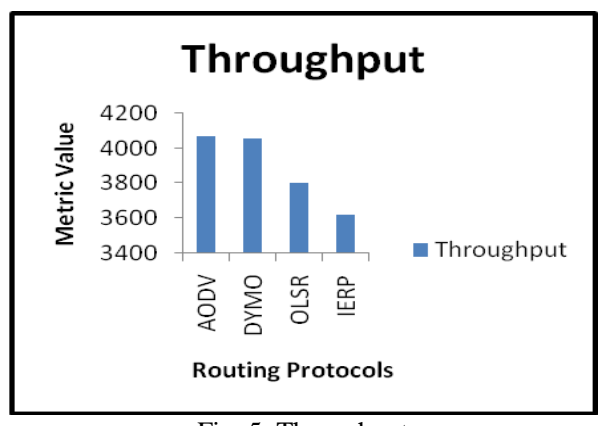

Fig. 5. Throughput

B. End to End Delay

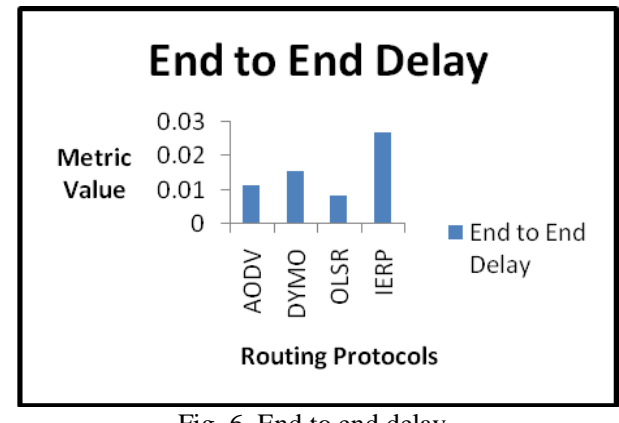

End to end delay refers to the time taken for a packet to be transmitted across a network from source to destination. Usually a data packet may take few extra second to reach the client or the server's end, which happens due to congestion in the communication network in the situation of a queue or when different routing paths are chosen by the routing protocol [9]. The graph below shows the end to end delay is greatest in IERP as compared to the others which are very small.

\section{Jitter}

Jitter is the variation in delay by different data packets that reached the destination and can seriously affect the quality of audio/video and thus an unwanted parameter [10]. Here we can see that the average jitter is fairly high in the case of IERP, then, it is DYMO, AODV [8] and least is in the case of OLSR.

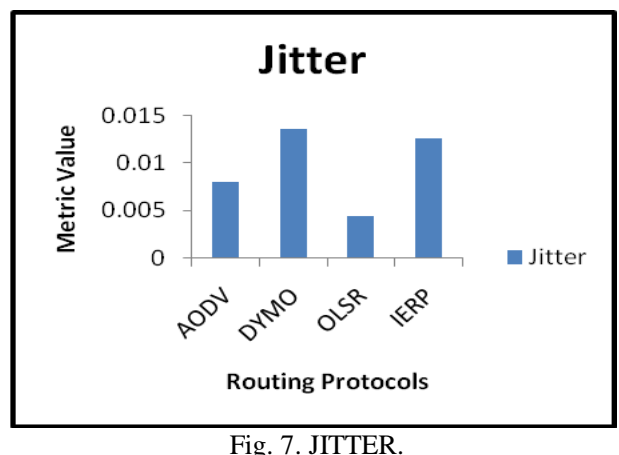

\section{Packet Delivery Ratio}

Packet delivery ratio is the fraction of packets sent by source that are received by the destination and is calculated by dividing the number of packets received by the 
destination through the number of packets originated by the application layer of the source [11]. Its higher value indicates good performance of the protocol. The graph below shows the best PDR is in the case of AODV and DYMO as compared to OLSR and IERP.

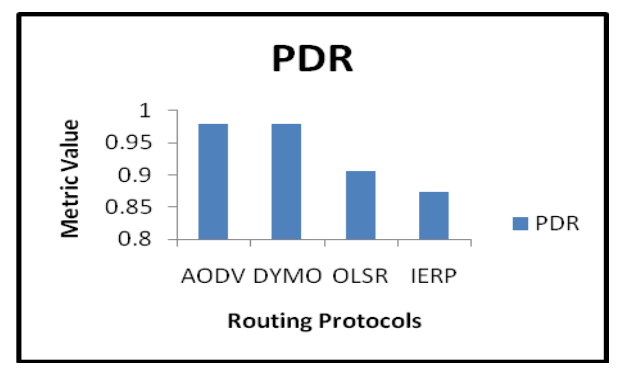

Fig. 8. Packet delivery ratio.

\section{E. Total Number of Packets Received}

Total number of packets received at the destination. Its count tells us the total number of packets received out of total number of packets sent, in this case 100 data packets were sent. The graph shows the best protocol to deliver the data packets to the destination are AODV and DYMO in comparison to OLSR and IERP.

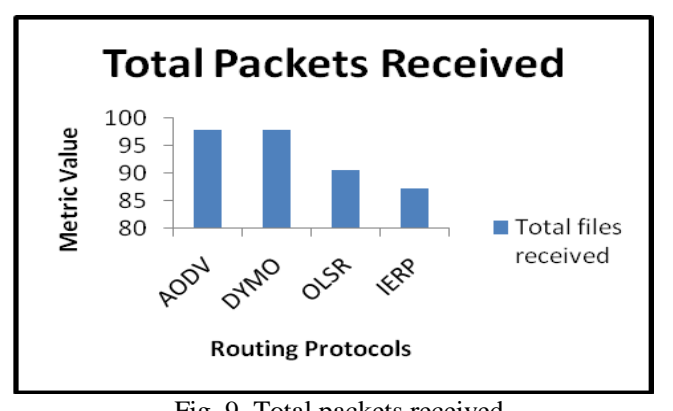

Fig. 9. Total packets received.

\section{CONCLUSION}

The above results give us a combine and comparative study of three types of protocols namely: Reactive, Proactive and Hybrid. Reactive protocols being: AODV and DYMO. OLSR (proactive protocol) and IERP (hybrid protocol) [12]. Here we can see that the reactive protocols are best in throughput, PDR and total packets received. While comparing the results of AODV and DYMO communication routing protocols individually, DYMO comes out to be the better one. Hybrid protocol IERP has high end to end delay and jitter values and the proactive protocol, OLSR stays second in the above mentioned performance parameters.

\section{REFERENCES}

[1] S. Mittal and P. Kaur, "Performance comparison of AODV, DSR and ZRP routing protocols in MANET's," in Proc. 09 International Conference on Advances in Computing, Control and Telecommunication Technologies, IEEE 2009.

[2] D. Oliver and Jorg, "Performance comparison of MANET routing Protocols in different network sizes," Institute of computer Science and applied mathematics computer Networks and distributed systems University of Berne, Switzerland IAM RVS February 2004 computer science projects.

[3] I. Chakeres and C. Perkins, Dynamic MANET on demand (DYMO) routing protocol, Internet- Draft Version 06, IETF, October 2006.

[4] E. Royer and C. Toh, "A review of current routing protocols for Adhoc mobile wireless networks," IEEE Personal Communications, vol. 6, issue 2, pp. 46-55, April 1999.

[5] B. Ahmed, M. Islam, and J. Rahman, "Simmulation, analysis and performance comparison among different routing protocols for wireless sensor networks," Computer Engineering and Intelligent Systems, ISSN 2222-1719, vol. 2, no. 4, 2011.

[6] Z. Haas, M. Pearlman, and P. Samar, "The interzone routing protocol (ierp) for ad hoc networks," draft-ietf-manet-zone-ierp-01.txt

[7] K. Navidi, W. Camp, and W. Patterson, "Discovering variables that affect MANET protocol performance," in Proc. GLOBECOM '07, IEEE Publication Date: 26-30 Nov. 2007.

[8] V. Thapar, B. Jain, and V. Sahni, "Routing protocols using random waypoint mobility model in wireless sensor networks," International Journal on Computer Science and Engineering, vol. 3, no. 8, pp. 3059, August 2011.

[9] P. Goyal, S. Vijay, and D. Jhariya, "Simulation and Performance Analysis of Routing Protocols in Wireless Sensor Network using Qualnet," International Journal of Computer Application, vol. 52, no. 2, August 2012.

[10] N. Bisht, R. Mishra, and R. Dhillon, "Mobility based performance analysis of wireless sensor networks," International Journal of Computer, Electronics \& Electrical Engineering, vol. 2, issue 2.

[11] P. Nand and S. Sharma, "Routing load analysis of broadcast based reactive routing protocols AODV, DSR and DYMO for MANET," International Journal of Grid and Distributed Computing, vol. 4, no. 1, pp. 81-92, March 2011.

[12] M. Amin, M. Abrar, Z. Khan, Abdusalam, and S. Rizwan, "Comparison of OLSR \& DYMO routing protocols on the basis of different performance metrics in mobile Ad-hoc networks," American Journal of Scientific Research, issue 37, pp. 34-57, 2011.

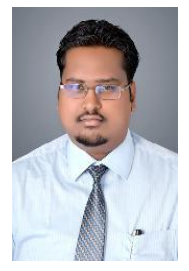

Ashish Allen Roberts is a research scholar and pursuing his M.Tech. Degree in Communication System Engg. in the department of Electronics \& Communication Engg., SHIATS-DU, Allahabad. His present research area is Wireless Communication \& Sensor Networks.

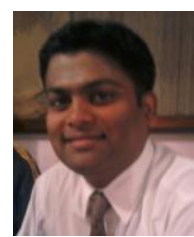

Ashish Xavier Das is a PhD Scholar in Electronics and Communication Engineering SHIATS-DU, Allahabad. $\mathrm{He}$ received his B.Tech. degree from AAIDU, Allahabad and M.Tech from SHIATS-DU, Allahabad. His research is focused on Routing Protocols in WSN. 